\title{
A Novel Foil Flip-Over System as the Final Layer in Wound Closure: Excellent Cosmetic Results and Patient Comfort
}

\author{
Eva Barbara Deerenberg, MD, * Hedwig Josephine Goyen, ${ }^{*}$ Ruth Kaufmann, MD,* \\ Johannes Jeekel, MD, PhD, ${ }^{\dagger}$ and Kai Munte, $\mathrm{MD}^{\ddagger}$
}

BACKGROUND Wound closure after excision is commonly done with sutures or staples. A new sutureless innovative wound closure system is available for sutureless skin closure.

OBJECTIVE To evaluate wound healing, patient comfort, and cosmetic results of a foil flip-over system for excision of small skin lesion.

MATERIALS AND METHODS Patients presenting to the department of Dermatology of Erasmus University Medical Center, Rotterdam, The Netherlands for skin surgery during a 1.5-year period were prospectively studied. Key outcome measures were wound healing, patient comfort, and cosmetic results. Three independent physicians scored photographs of the scars. Evaluation tools used were comfort and body image questionnaires and visual analogue scales.

RESULTS Ninety-six patients with 103 lesions were included in our study. The surgeon scored wound healing as excellent or good in $96 \%$. No wound infections occurred. Ninety-two percent of patients scored removal of the system as comfortable. Median patient grade of scar after 1 month was 8 out of 10 points (interquartile range [IQR] 7-9). Median independent physician grade of photographs of the scars was 7.7 (IQR 7.1-8.0).

CONCLUSION Sutureless foil flip-over is promising, with excellent patient comfort characteristics and good to excellent cosmetic results.

Eurotap BV, Soest, the Netherlands, provided the OptiClose System free of charge for this study.

$\mathrm{T}$ housands of patients undergo surgical excision of skin lesions each year. In 2001, more than 1.4 million skin excisions were performed in the United States, and the number of procedures is increasing. ${ }^{1-3}$ After excision of a small skin lesion, the wound needs to be closed. For the majority of excisions of lesions, subcutaneous and subsequent transcutaneous or subcuticular sutures are used to relieve tension on the edges of the wound and to approximate the wound edges. ${ }^{4,5}$ Although wound closure with sutures is effective, it requires specialized instruments and is time consuming. Furthermore, results are operator dependent and a subsequent visit for suture removal is required.
Other disadvantages of cutaneous sutures are risk of foreign-body reaction, risk of bacterial migration into the wound bed, and patient discomfort during removal of the sutures. ${ }^{6,7}$ To optimize surgical handling characteristics, patient comfort and cosmetic results, the OptiClose System, consisting of an excision foil and a flip-over-strip wound closure system, was developed. The excision foil keeps the wound area sterile and protected during excision. The flip-over strip system provides fast, accurate, easy closure of the skin. The system promises comfort during wearing and pain-free removal. The aim of our study was to evaluate the wound healing, patient comfort, and

\footnotetext{
$*$ Departments of Surgery; ${ }^{\dagger}$ Neuroscience; ${ }^{\star}$ Dermatology, ErasmusMC University Medical Center, Rotterdam, The Netherlands
} 
cosmetic results of this excision foil flip-over system (FFS).

\section{Materials and Methods}

\section{Patients}

A prospective cohort study at the department of Dermatology at the ErasmusMC University Medical Center, Rotterdam, the Netherlands, was performed between December 2009 and May 2011. Approval was obtained from the Medical Ethical Review Board of the ErasmusMC University Medical Center. All patients referred for excision of skin lesions smaller than $3 \mathrm{~cm}$ on the trunk or the limbs were evaluated for eligibility and included in our cohort. Patients with an excision margin of the lesion $>3 \mathrm{~cm}$, with lesions in the face area, or undergoing Mohs surgery were excluded, as were patients with known allergy to any kind of wound foil. Before surgery, a photograph was taken of the lesion.

\section{Foil flip-over system}

The FFS is shown in Figure 1. After disinfecting the operating field twice, the FFS was applied. Excision of the lesion was performed through the excision foil of the FFS. After the procedure was completed, the subcutaneous tissue was closed with subcutaneous slowly absorbable monofilament sutures, (polydioxanone 3.0 or 4.0 ). Then the excision foil of the FFS was removed, revealing the flip-over system, which allowed approximation of the wound edges by securing two contralateral flip-over strips to the opposed skin. The flip-overs do not cover the whole wound area, allowing exudate to discharge. Step-by-step use of the FFS is shown in Figure 2.

\section{Follow-up}

Ten to 14 days after surgery, patients returned to the outpatient clinic of the Department of Dermatology for removal of the FFS. After removal of the FFS, a photograph of the scar was taken. The surgeon filled in a questionnaire about wound condition, approx-

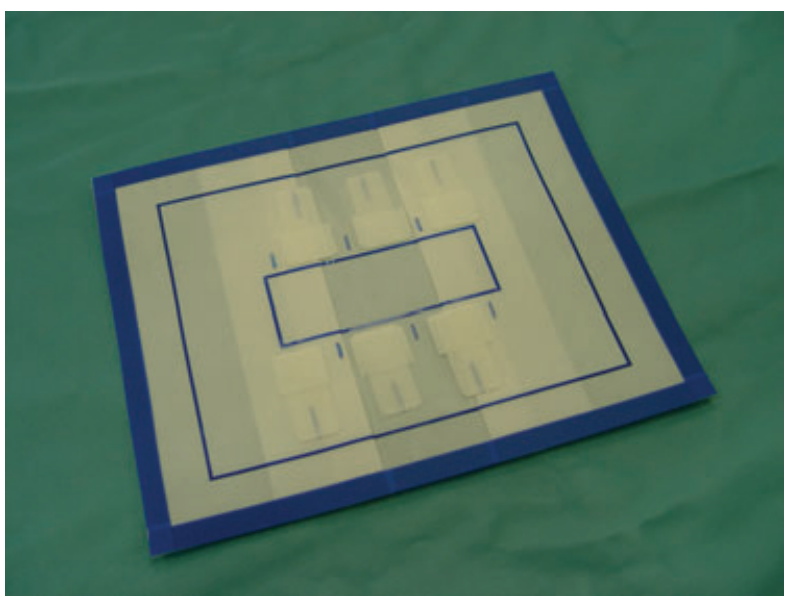

Figure 1. Foil flip-over system (FFS; OptiClose System).

imation of the wound edges, ease of removal and patient comfort during removal of the product, and any adverse reactions. Directly after removal of the system, patients scored discomfort on a visual analogue scale (VAS) from 0 to 100 . Patients received a comfort questionnaire within 1 month after surgery to evaluate comfort during wearing and removal of the FFS. They scored the cosmetic result of the scar with a grade from 0 to 10 . Patients received a body image questionnaire 6 months after surgery containing questions concerning their satisfaction with the operation and scar and scored the cosmetic result of the scar with a grade from 0 to 10 . Photographs of the lesion and the scar after removal of the FFS were collected in a database. The combination of photographs of the lesion and the scar were in random order presented on a website to three independent physicians (a dermatologist, a general surgeon, and a plastic surgeon). Each physician graded the wound condition and approximation on a VAS ranging from 1 to 10 in an individual online session. After grading, it was not possible to return to an earlier photograph or change the grade. Examples of photographs are shown in Figure 3.

\section{Statistical analysis}

Wound approximation, comfort during wearing and removing, grading of the scars, relationship between location and grade, and relationship between comorbidities and complications were analyzed using 

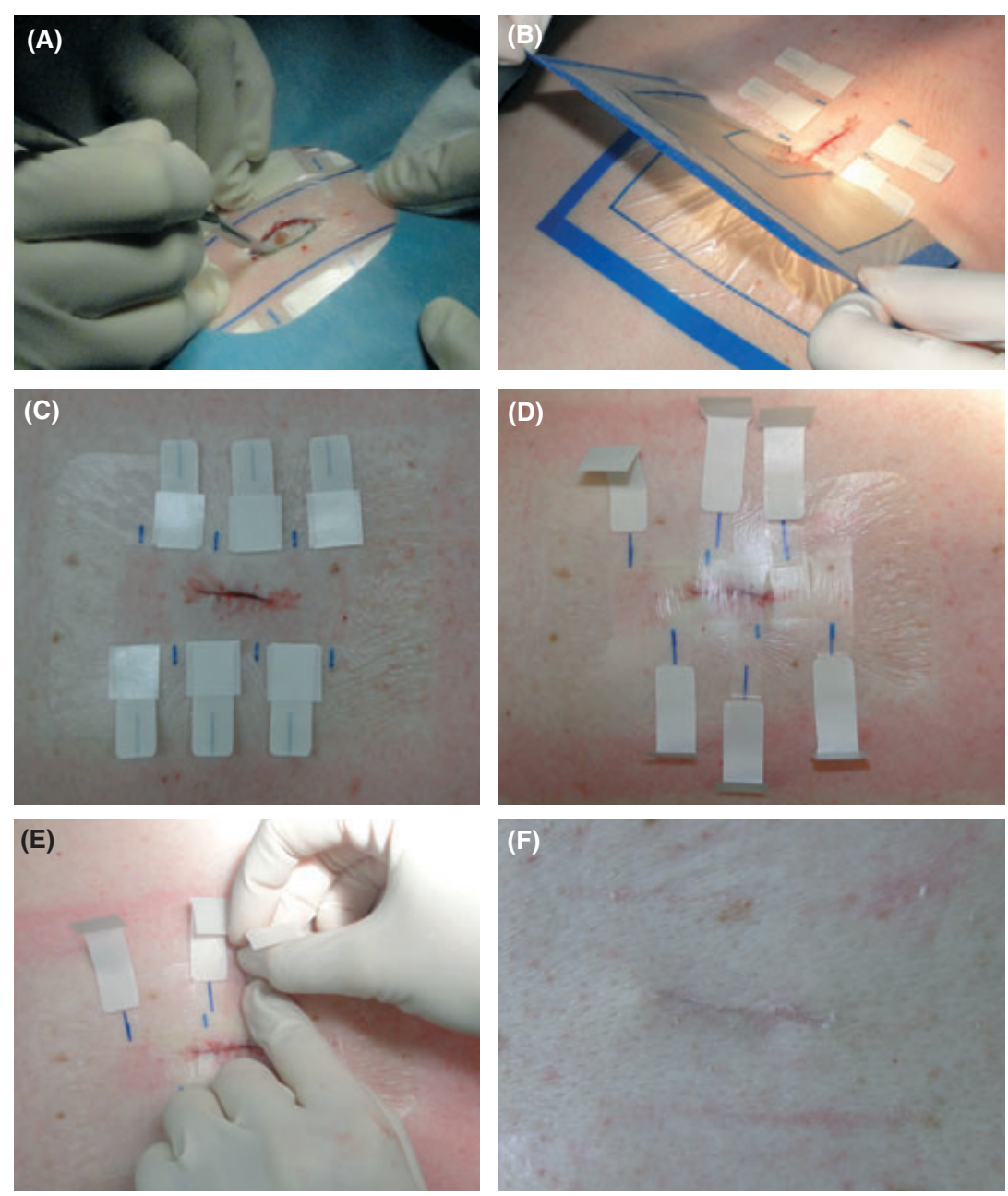

Figure 2. Foil flip-over system use. Step-by-step photographs: (A) Excision through FFS. (B) Removal of excision foil. (C) Flip-over system. (D) Securing contralateral flip-over strips to opposed skin. (E) Easy removal of pull-strips. (F) Wound approximation after removal of FFS (14 days after excision).
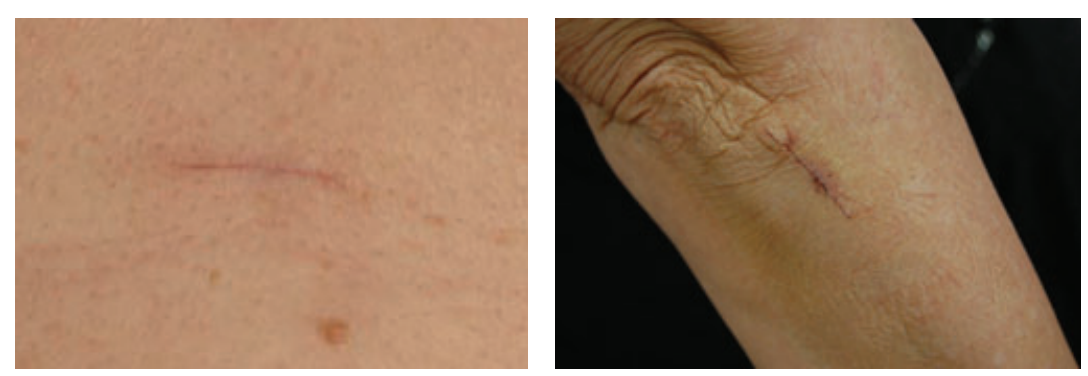

Figure 3. ( $A$ and B) Examples of scored photographs of scars.

nonparametric tests (Kruskal-Wallis, Mann-

Whitney, chi-square, Fisher exact) because the data did not have a normal distribution. All results were presented using medians and interquartile ranges (IQR). Exact methods for significance were used when computational limits allowed it. All reported $p$-values are two-sided and considered significant if $<.05$. Statistical analysis was performed using PSAW statistical software package, version 17 (IBM SPSS statistics, Armonk, NY). 


\section{Results}

Ninety-six patients with 103 lesions were included in our study. Patient characteristics are presented in Table 1. Most excised lesions were located on the back $(44 \%)$ and chest $(26 \%)$. One patient died 1 month after excision of the lesion from cardiovascular disease and did not complete followup. Although $27 \%$ of patients had a risk factor for wound infection (diabetes, smoking, or use of immunosuppressive drugs), no wound infection occurred.

In all patients, the FFS was removed at the outpatient clinic. In 97 cases, the surgeon responded to the questionnaire (94\%) after removal of the FFS. In two patients, redness (without exudate of pus) was seen, and in two patients a hematoma was found. In seven patients $(7 \%)$, an allergic reaction was found; three patients had a mild reaction with erythema and edema, three patients had vesicles; and in one patient, a severe allergic reaction with bulla was found (Figure 4). Despite the allergic reactions, wound healing in these patients was excellent or good, and the reaction disappeared quickly after removal of the foil. The surgeon scored healing and approximation of the wound edges as excellent in $69 \%$, good in $27 \%$, moderate in $2 \%$, and bad in $2 \%$ of lesions. Eighty-three percent of patients returned the comfort questionnaire. The FFS adhered well to

\begin{tabular}{|c|c|}
\hline Characteristic & Value \\
\hline \multicolumn{2}{|l|}{ Patient ( $n=96)$} \\
\hline Male:female, $n$ & $57: 39$ \\
\hline Age, mean $\pm \mathrm{SD}$ (range) & $58 \pm 18.0(14-87)$ \\
\hline Smoker, $n(\%)$ & $13(14)$ \\
\hline Diabetes mellitus, $n(\%)$ & $7(7)$ \\
\hline $\begin{array}{l}\text { Use of immunosuppressive } \\
\text { drugs, } n(\%)\end{array}$ & $6(6)$ \\
\hline \multicolumn{2}{|l|}{ Lesion, $n(\%)(n=103)$} \\
\hline Chest & $27(26)$ \\
\hline Back & $45(44)$ \\
\hline Abdomen & $8(8)$ \\
\hline Upper extremity & $16(15)$ \\
\hline Lower extremity & $7(7)$ \\
\hline
\end{tabular}

the skin in $80 \%$ of patients until removal of the FFS at the outpatient clinic. Eighty-two percent of patients felt comfortable wearing the FFS, although $18 \%$ reported complaints of discomfort or slight itching. Feelings of discomfort or itching were reported significantly more when the FFS was used on the back $(p<.05)$. Ninety-two percent of patients judged removal of the FFS from not to just a little uncomfortable. Patients graded the cosmetic result of their scar after 1 month with a median 8 out of 10 points (IQR 7-9). Adherence of the foil to the skin, comfort during wearing, and grade of the scar were not related to location of the excised lesion.

The body image questionnaire was received back in $81(78 \%)$ of 103 cases. After 6 months, the majority of patients were very satisfied $(36 \%)$ or satisfied $(38 \%)$ with their scar, and $6 \%$ were not satisfied with the cosmetic results. Patients graded their scar after 6 months with a median 8 out of 10 points (IQR 7-9). The degree of satisfaction was the lowest for patients with a scar on the chest $(p<.05)$. The perception that excision of the lesion damaged the body was lower in patients with excision of a lesion on the back $(p<.05)$. The median grade of the photographs of the 103 scars from three independent physicians was 7.7 (IQR 7.1-8.0, (range 3.88.8). Median grades per location were chest 7.8 (IQR 7.2-8.2), abdomen 7.8 (IQR 7.4-8.6), back 7.6 (IQR 7.1-8.0), upper extremities 7.5 (IQR 7.0-

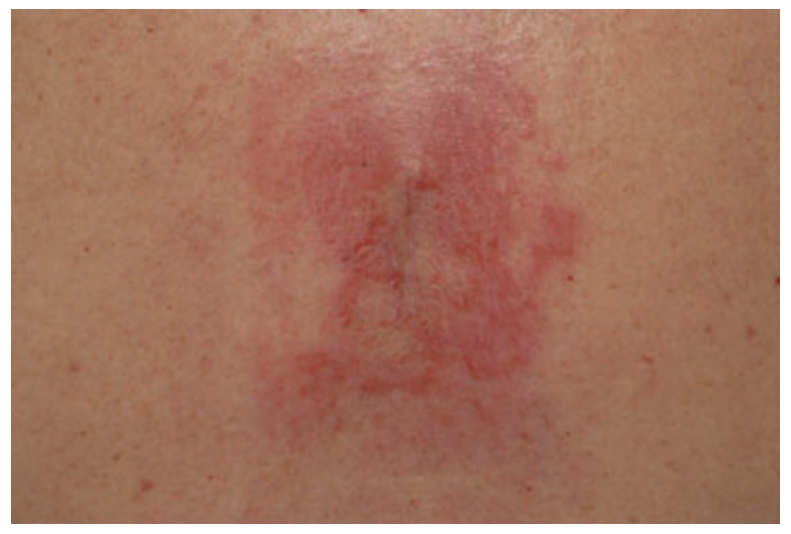

Figure 4. Photograph of allergic reaction with excellent wound approximation. 
7.9), and lower extremities 7.5 (IQR 6.8-7.9). The mean grades of the scars by the independent physicians were not significantly different between the different locations on the body.

\section{Discussion}

The ideal method of wound closure is reliable, comfortable, time efficient, and inexpensive and produces optimal cosmetic outcome. ${ }^{8}$ The purpose of this prospective cohort study was to evaluate these specific characteristics of the FFS. Wound dehiscence is the most significant factor in effective wound closure. ${ }^{4}$ Subcutaneous sutures prevent wound dehiscence in large part, but the method of closure of the top layer of the skin contributes as well. Our study showed good to excellent wound healing in $96 \%$ of patients and no wound dehiscence. The FFS adhered very well to the skin in four out of five patients. Good wound healing is generally seen with wound foils. In four small randomized studies $(N=107)$ with some type of sutureless wound closure foil, minor wound dehiscence was reported in one patient that resolved with local wound care. ${ }^{4,8-10}$ Concerns or results regarding wound foil adherence were not reported in any of these studies.

Eighty percent of patients in our study felt comfortable wearing the FFS, and removal of FFS was in the vast majority of the patients not or only a little uncomfortable. This is an advantage over cutaneous sutures or staples that need (often uncomfortable) removal. The comfort of wound foil is comparable with that of subcuticular sutures, ${ }^{11}$ but subcuticular sutures take longer to place than the FFS.

Four small randomized studies compared a different type of wound closure film with subcuticular or running cutaneous sutures for cosmetic results. ${ }^{4,8-10}$ In the study of Grottkau et al., the surgeons scored the cosmetic appearance of the scars using a VAS. They found no significant differences between cosmetic results after wound closure with foil or sutures. ${ }^{8}$ Kerrigan and colleagues assessed the cosmetic results with the visual assessment tool of linear scars. Patients preferred the quality and appearance of the scars after wound closure foil. The surgeons graded the same scars, but they preferred the scars of the wounds closed with sutures. ${ }^{9}$ In our study, the patients and physicians were very satisfied with the cosmetic results after wound closure foil, grading the scars with scores of 8 and 7.7 out of 10 points. These grades exceed expectations, based on other studies. ${ }^{4,8-10}$

Data from the body image questionnaire showed that patients with scars on the back felt least damaged and patients with scars on the thorax felt most damaged after excision. This outcome is most likely not related to the use of FFS but to the location of the scar. The more visible the scar is, the more damaged the patient feels. In patients in whom the scar is more visible or who are aesthetically more demanding, sutures may be a better choice for skin closure, because the epidermis can be more finely approximated using sutures.

The use of sutureless wound foil appears to be a time-efficient solution for wound closure after surgery. Significantly shorter wound closure time has been reported in several studies, although study results are difficult to compare, because of heterogeneity in tests used. ${ }^{4,8-10}$ In our study, wound closure time was not measured, but a decrease in closure time is assumed.

The use of the FFS is cost effective. The wound closure foil is in the same price range as one polydioxanone or polypropylene suture, which makes the FFS cost equivalent to traditional twosuture closure. Total costs are lower than with final layer closure with nonabsorbable sutures because an extra visit to the outpatient clinic is required for removal of the latter. The patient can remove the wound closure foil, which saves the cost of this extra visit to the outpatient department. However, regarding outpatient department visits, there is no cost benefit of the FFS over closure with absorbable sutures. Operating time is shorter than with subcuticular sutures because of the ease of wound closure 
with the FFS. The amount of time saved is not relevant for a single patient, but becomes relevant when taking into account that thousands of patients undergo skin excision every year.

Dermatologic surgery is considered to be cleancontaminated surgery, which is defined as $5 \%$ to $15 \%$ postoperative infections. ${ }^{12}$ Reported postoperative infection rates in skin excisions vary from $0.7 \%$ to $2.1 \% .^{13,14}$ In our study, two cases of skin redness occurred in 103 excisions (1.94\%), but no wound infections were found. We found that $7 \%$ of the patients had a reaction to the FFS, including one severe allergic reaction. Only one other study found an allergic reaction to wound foil, but it was classified as an allergic reaction to bacitracin ointment and not to the sutureless wound foil. ${ }^{4}$ The FFS should not be used in patients with a history of tape or adhesive allergy.

Because our study was a prospective cohort study, patients were only treated with FFS. There is no comparison with other types of skin closure, such as staples, tapes, or adhesive foils. ${ }^{8,10}$ Patients and physicians were not blinded and might have had a bias toward other techniques of skin closure. Nevertheless, our prospective cohort study revealed promising cosmetic and patient comfort results.

\section{Conclusion}

Our study showed that OptiClose is an innovative, attractive, easy-to-use wound closure system with excellent wound healing, high patient comfort, and outstanding cosmetic results.

\section{Acknowledgments We thank Professor J. F.} Lange, MD, PhD (surgeon), I. Mathijssen, MD, PhD (plastic and reconstructive surgeon), and F.B. de Waard-van der Spek, MD, PhD (dermatologist) for grading all photographs as independent physicians. We thank Eurotape BV, Soest, the Netherlands, for providing the OptiClose Systems for this study.

\section{References}

1. Cullen KA, Hall MJ, Golosinskiy A. Ambulatory surgery in the United States, 2006. Natl Health Stat Report 2009;28(11):1-25.

2. Roenigk RK. Dermatologists perform more skin surgery than any other specialist: implications for health care policy, graduate and continuing medical education. Dermatol Surg 2008;34(3): 293-300.

3. Tuleya S. A status report: dermatological surgery.2002;10(1). Available from: http://www.skinandagingcom/article/620 Accessed January 15, 2002.

4. Kuo F, Lee D, Rogers GS. Prospective, randomized, blinded study of a new wound closure film versus cutaneous suture for surgical wound closure. Dermatol Surg 2006;32(5):676-81.

5. Vistnes L, editor. Basic principles of cutaneous surgery. In:pstein E, Epstein EJr, editors. Skin Surgery (6th ed). Philadelphia: WB Saunders; 1987. pp. 44-55.

6. Basha SL, Rochon ML, Quinones JN, Coassolo KM, et al. Randomized controlled trial of wound complication rates of subcuticular suture vs staples for skin closure at cesarean delivery. Am J Obstet Gynecol. 2010;203(3):285.e1-8.

7. Fick JL, Novo RE, Kirchhof N. Comparison of gross and histologic tissue responses of skin incisions closed by use of absorbable subcuticular staples, cutaneous metal staples, and polyglactin 910 suture in pigs. Am J Vet Res 2005;66(11): 1975-84.

8. Grottkau BE, Rebello G, Merlin G, Winograd JM. Coaptive film versus subcuticular suture: comparing skin closure time after posterior spinal instrumented fusion in pediatric patients with spinal deformity. Spine (Phila Pa 1976) 2010;35(23):2027-9.

9. Kerrigan CL, Homa K. Visual assessment of linear scars: a new tool. Plast Reconstr Surg 2009;124(5):1513-9.

10. Rebello G, Parikh R, Grottkau B. Coaptive film versus subcuticular suture: comparing skin closure time following identical, single-session, bilateral limb surgery in children. J Pediatr Orthop 2009;29(6):626-8.

11. Kerrigan CL, Homa K. Evaluation of a new wound closure device for linear surgical incisions: 3M Steri-Strip S Surgical Skin Closure versus subcuticular closure. Plast Reconstr Surg 2010;125 (1):186-94.

12. Bordeaux JS, Martires KJ, Goldberg D, Pattee SF, et al. Prospective evaluation of dermatologic surgery complications including patients on multiple antiplatelet and anticoagulant medications. J Am Acad Dermatol 2011;65(3):576-83.

13. Whitaker DC, Grande DJ, Johnson SS. Wound infection rate in dermatologic surgery. J Dermatol Surg Oncol 1988;14(5):525-8.

14. Futoryan T, Grande D. Postoperative wound infection rates in dermatologic surgery. Dermatol Surg 1995;21(6):509-14.

Address correspondence and reprint requests to: $\mathrm{EB}$ Deerenberg, MD, ErasmusMC, room Z-838, Postbus 2400, 3000 CA Rotterdam, The Netherlands, or e-mail: e.deerenberg@erasmusmc.nl 